\title{
NEW NUMERICAL METHOD TO STUDY PHASE TRANSITIONS AND ITS APPLICATIONS*
}

\author{
$\mathrm{ANL} / \mathrm{CP}--75038$
}

DE92 006995

\author{
J. Lee \\ Materials Science Division \\ Argonne National Laboratory \\ Argonne, IL 60439 \\ J. M. Kosterlitz \\ Department of Physics \\ Brown University \\ Providence, RI 02912
}

\begin{abstract}
The submitted manuscript has been authored by a contractor of the U. S. Government under contract No. W-31-109-ENG-38. Accordingly, the U.S. Government retains a nonexclusive, royalty-free license to publish or reproduce the publisheci form of this contribution, or allow others to do so, for U.S. Government purposes.
\end{abstract}

\section{NOVEMBER 1991}

\section{DISCLAIMER}

\footnotetext{
This report was prepared as an account of work sponsored by an agency of the United States Government. Neither the United States Gnvernment nor any agency thereof, nor any of their employees, makes any warranty, express or implied, or assumes any legal liability or responsibility for the accuracy, completeness, or usefulness of any information, apparatus, product, or process disclosed, or represents that its use would not infringe privately owned rights. Reference herein to any specific commercial product, process, or service by trade name, trademark, manufacturer, or otherwise does not necessarily constitute or imply its endorsement, recommendation, or favoring by the United States Government or any agency thereof. The views and opinions of authors expressed herein do not necessarily state or reflect those of the United States Government ol any agency thereof.
}

Submitted to AIP Conference on Computer Aided Studies in Statistical Physics, Taiwan, Iune 20-26, 1991.

*Work Supported by the U.S. Department of Energy, BES-Materials Sciences. under Contract W-31-109-ENG-38.

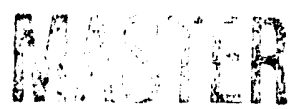

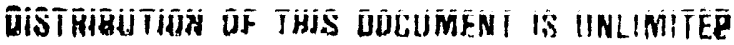




\title{
NEW NUMERICAL METHOD TO STUDY PHASE TRANSITIONS AND ITS APPLICATIONS
}

\author{
Jooyoung Lee \\ Material Science Division, Argonne National Laboratory, \\ Argonne, IL60439, USA \\ J. M. Kosterlitz \\ Department of Physics, Brown University, \\ Providence, RI 02912, USA
}

\begin{abstract}
We present a powerful method of identifying the nature of transitions by numerical simulation of finite systems. By studying the finite size scaling properties of free energy barrier between competing states, we can identify unambiguously a weak first order transition even when accessible system sizes are $L / \xi<0.05$ as in the five state Potts model in two dimensions. When studying a continuous phase transition we obtain quite accurate estimates of critical exponents by treating it as a field driven first order transition. The method has been successfully applied to various systems
\end{abstract}

\section{INTRODTJCTION}

There ure not many statistical models which can be solved analytically with non trivial solurions. With rapio development of computer technology, computer simulation methods appear to be a realistic substitute for theoretical solutions which may not be available at all.

Mathematically speaking there occur no phase transitions in finite systems and the success of computer simulations near phase transitions depends on the finite size scaling, ${ }^{1-3}$ where we extrapolate to the thermodynamic limits $(N$, $V \rightarrow \infty$ ) from small system sizes. This paper will describe how one can extend the idea of finite size scaling into the task of identifying the nature of transition. As a by-product it, in case of continuous transitions, we also get a surprisingly accurate way to obtain correlation-length exponent $\nu$ and critical temperature $T_{\mathrm{c}}$.

The philosophy of all the existing methods for identifying the nature of transition relies on the outcomes of the finite size scaling of first order transitions with system size $L$ much larger than the correlation length $\xi$, which remains finite at first order transitions contrary to the second order. Therefore it is not surprising that they fail to give unambiguous answer (even with large size simulations ${ }^{4-6}$ up to $L=256$ ) to ider tifying weak first order transitions like $q=$ 5 Potts model ${ }^{7}$ in two dimensions, where $\xi>1000$ lattice spacings. ${ }^{4}$ For example, 
the hysteresis effect, which has been the most popular method, comes from the competition between observation time and equilibration time in computer simulations of a finite size system. At a very strong first order transition the equilibration time near $T_{c}$ can be much larger than the order of observation time, even though the latter can be as long as allowed by CPU time. One might be able to identify the nature of transition correct from a very careful analysis of the hysteresis effect, ${ }^{8-10}$ but as the transition becomes weaker the effect becomes more ambiguous.

Recently ${ }^{11}$, Binder has introduced a quantity that is believed to be very sensitive to the nature of transition. For energy it is defined as

$$
V_{L}=1-\frac{<E^{4}>_{L}}{3<E^{2}>_{L}^{2}}
$$

With a continuous transition, $V_{L} \rightarrow 2 / 3$ for all temperature as $L \rightarrow \infty$. For a first order transition it takes on the value $2 / 3$ for high and low temperature, but in a temperature range of $O\left(L^{-d}\right)$ of $T_{c}$ it can take on the value less than $2 / 3$. Finite size scaling of the first order transition predicts $\left.V_{L}\right|_{\min }$ up to $O\left(L^{-d}\right)$ as ${ }^{12}$

$$
\left.V_{L}\right|_{\min }=2 / 3-\left(e_{1} / e_{2}-e_{2} / e_{1}\right)^{2} / 12+A L^{-d}
$$

which correct some earlier work. ${ }^{13}$ Here $e_{1}$ and $e_{2}$ are bulk energy value for two competing states and $A$ is a rather complicated expression involving $T_{c}$, $e_{1}, e_{2}$ and bulk specific heat of each phase. In principle $\left.V_{L}\right|_{\min }$ can be used to distinguish between continuous and first order (temperature driven) transition. but we find ${ }^{12}$ that the asymtotic approach of $L^{-d}$ sets in only for $L \gg \xi$ and more over $\left.V_{L}\right|_{\min }=0.66622$. for the case of $q=5$ Potts model in two dimensions which is quite close to $2 / 3$ to be distinguished numerically.

When one is forced to a numerical investigation on a totally unknown system, the first thing he has to do is to identify its nature, which dictates the next step. With a first order transition one may wants to evaluate, critical temperature, correlation length, discontinuities in various quantities, etc., whereas with a continuous transition, various exponents, etc. Therefore, without information on the nature of the transition, it makes little sense to interd to obtain critical exponents which is relevant only to continuous transitions.

\section{FINITE SIZE SCALING OF BULK FREE ENERGY BARRIER ${ }^{14}$}

Distinguishing a weak first order transition from continuous transitions is one of the ultimate tasks that computer simulation methods would like to provide. To do better than what has been done in the literature, it is obvious that we need to investigate the origin of the first order transitions consequence of which makes them so much different from continuous transitions when $L \gg \xi$. The essential feature of a discontinuous transition is that there exist a set of competing bulk phases with infinite free energy barriers of $O\left(L^{d-1}\right)$ between 
them in the thermodynamic limits. Rather than studying the outcome of this large free energy barrier (which gives rise to the results of finite size scaling of strong first order in the limit $L \gg \xi$ ), we want to focus on how this barrier will evolve as a function of system size depending on the nature of the transition.

The key idea is, taking Ising model in two dimensions as an example of field-driven first order transition, how the two delta-function-like distribution of magnetization at $m= \pm m_{0} \neq 0$ below $T_{c}$ develops to a single gaussian centered at $n=0$ as the temperature is increased through $T_{c}$. First, we consider a case of strong (field driven) first order transition with $L \gg \xi$. For Ising model this can be realized simply with $T \ll T_{c}$ in zero magnetic field $h=0$. In this limit, the bulk free energy of restricted magnetization can be expanded as power of $1 / L$ as follows. ${ }^{15,16}$

$$
F(m, T, L)=L^{d} f_{0}(m, T)+L^{d-1} f_{1}(m, T)+O\left(L^{d-2}\right)
$$

Note that the form of $L^{d-1}$ in Eq. 3 is relevant for systems with a discrete symmetry of the ground states such as Potts models. In case of a system with continuous symmetry of the ground states such as XY or Heisenberg model it is replaced by $L^{d-2}$. The bulk free energy density $f_{0}$ is minimum and constant for $-m_{0} \leq m \leq m_{0}$ and the interfacial term $f_{1}$ has maximum at $m=0$ due to the \pm symmetry of the Ising model. Then it is obvious that $F$ has minima at $m_{1}(L)=-m_{0}-O\left(L^{-1}\right)$ and $m_{2}(L)=m_{0}+O\left(L^{-1}\right)$ with a barrier of

$$
\Delta F(t, L)=F(0, t, L)-F\left(m_{1}, t, L\right) \sim A(t) L^{d-1}+O\left(L^{d-2}\right)
$$

Therefore we find the known result of symmetry breaking below $T_{c}$ that, even though there are two equivalently most probable states, once the system sits on one of them it is not allowed to reach the other equivalent state by infinite barrier of $O\left(L^{-d}\right)$.

Here, $t=T / T_{c}-1$ drives the system along the field driven first order transition line at $t<0$ through the critical point at $t=0$ into the disordered phase $t>0$. For a temperature driven first order transition the roles of $h=0$ and $m$ are played by $T_{c}$ and internal energy $E$. There may be a field $g$ which drives the system along the temperature driven first order transition line at $g<0$ through the critical or multicritical point at $g=0$ into the second order transition line $g>0$. For two dimensional Potts model $g<0$ for $q>4$ and $g=0$ for $q=4$. Therefore $F(E, g, L)$ is the equivalent quantity of $F(m, t, L)$ in Eq. 3.

When $L \ll \xi, F(X, g, L)$ is dominated by its singular part and finite size scaling theory tells us that it may be written in terms of scaling variables $x=X L^{\lambda}$ and $y=g L^{\lambda_{\theta}}$ as $F(X, g, L)=B(x, y) \cdot{ }^{16,17}$ For small $x$ and $y$, $B(x, y)$ has an analytic expansion in $x$ and $y$. At a first order transition, $y<0$, $B(x, y)$ must have a set of minima of equal depth corresponding equivalent states, at $x_{i}=x_{i}(y)$. Two local minima guarantee that there exist at least one 
maximum (or saddle point) at $x_{m}=x_{m}(y)$, which corresponds to the configuration with maximum interface between competing states. So that the barrier between equivalent local minima $\Delta F(y) \equiv B\left(x_{m}(y), y\right)-B\left(x_{i}(y), y\right)$ will grow with increasing $-y$, eventually crossing over to the strong first order behavior of $L^{d-1}$.

The simplest possible scenario, which holds for simple systems like ferromagnetic $q$-state Potts models with continuous transitions, is with $y=t L^{1 / \nu}$ and $x=M L^{\beta / \nu}$. Here one can show explicitly that for small $x$ and $y$

$$
\Delta F(t, L) \simeq a-b t L^{1 / \nu}
$$

With periodic boundary conditions and $L^{d}$ cubic geometry of the system, $a$ and $b$ are positive constant independent of $L$ and they depend on boundary conditions and the geometry of the system. An assumption has been made here that the system size $L$ is sufficiently large so that all the irrelevant variables may be ignored. Given this, it follows that $\Delta F(g, L)$ increases with $L$ in the first order regime $(g<0)$, is an $L$-independent constant (possibly zero) at the critical point $(g=0)$ and decreases with $L$ in a continuous transition (or disordered) regime $(g>0)$. This general argument constitute a very sensitive test of the nature of a transition even with $L<\xi$. Rather than dependirig on the quantitative estimate of thermodynamic values as $L \rightarrow \infty$, such as Binder's cumvilant or exponent $d$ of specific heat and susceptibility, one can determine the nature of a transition from the qualitative behavior of $\Delta F(g, L)$ which is the characteristic of the type of transition. However a note of cation is needed here. One can safely argue that the transition is of first order if $\triangle F(g, L)$ is found to increase with $L$, but with $a=0$, if no peak structure is seen, it may merely mean that $L$ is too small for the peak to be noticeable above numerical noise or that irrelevant variables have not scaled out. In this case one needs to seek how to increase $a$ without affecting the critical properties of the model

\section{TEMPERATURE DRIVEN FIRST ORDER TRANSITIONS}

To study temperature driven first order transition, one simply need to simulate a given system at near phase transition accumulating the histogram of internal energy, which is the conjugate to the temperature. For the case of pressure driven first order transition one need the histogram of volume, which is the conjugate to the pressure. In $N$ Monte Carlo (MC) sweeps, standard probability theory implies that the number of times the energy $E$ is realized is

$$
H(E, \beta, L)=N Z^{-1}(\beta, L) \Omega(E, L) \exp (-\beta E) \equiv \exp \{-A(E, \beta, L)\}
$$

where $\Omega(E, L)$ is the number of states with energy $E$ and $Z(\beta)$ is the partition function. $A(E, \beta, L)$ differs from the bulk free energy $F(E, \beta, L)$ by a temperature- and $N$-dependent additive quantity. Therefore, at fixed $\beta, L, N$ 


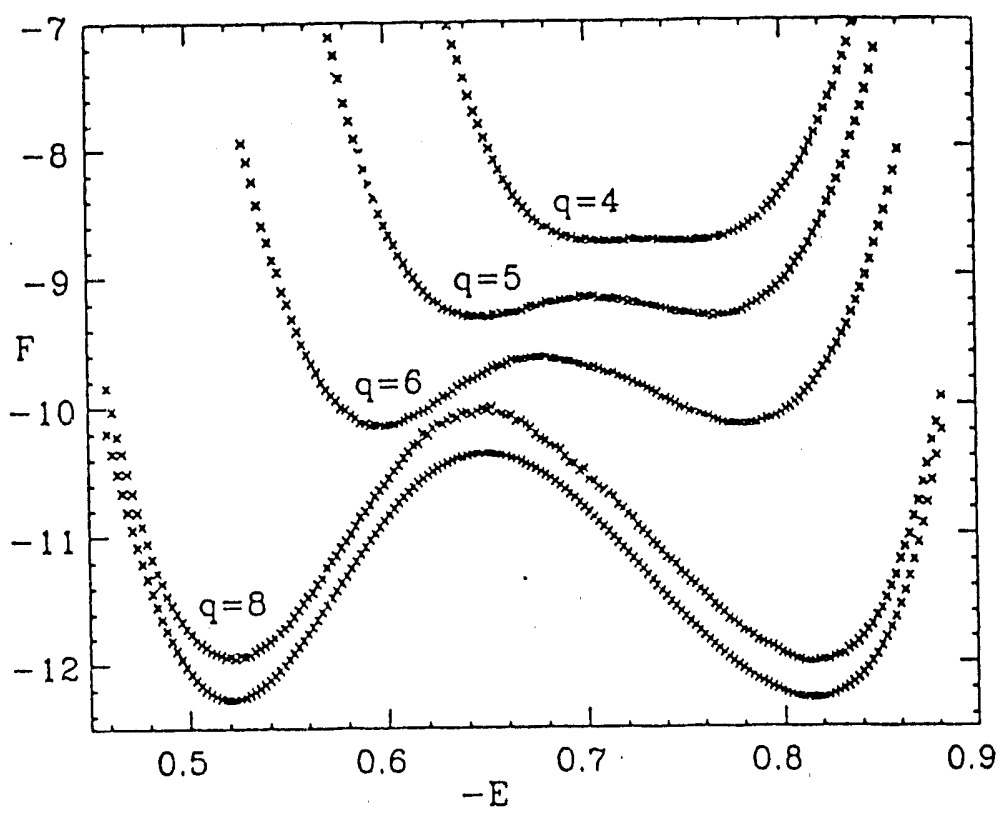

Fig. 1. $A\left(E, \beta_{c}(L), L\right)$ for $q=4,5,6,8$ Potts models for $32 \times 32$.

Bottom curve is $q=8$ data smoothed by polynomial fit. The energy scale is normalized to unity for complete order.

the shape of $A(E)$ will be same to that of $F(E)$ and we immediately obtain the crucial result

$$
\Delta F_{E}(L)=\Delta A_{E}(L) \equiv A\left(E_{1}, \beta_{c}, L\right)-A\left(E_{m}, \beta_{c}, L\right)
$$

where the function $A^{\prime}(E, \beta, L)$ have minima of equal depth at $E=E_{1}, E_{2}$ and maxima at $E_{m}$, at the pseudo-critical temperature $\beta_{c}(L)$ corresponding to the coexistence of ordered and disordered states. Eq. 7 is important since a measurement of $\triangle A$ from simulation gives a direci evaluation of the corresponding $\Delta F$. The technical problem of computing $A(E, \beta, L)$ at the transition has been solved by the histogram method as used by Ferrenberg and Swendsen ${ }^{18}$ who demonstrated how to extrapolate data from one value of $\beta$ to nearby values. Since such extrapolations are accurate only for $\delta \beta \sim O\left(L^{-d}\right)$ near a first order transition, one needs to locate $\beta_{c}(L)$ reasonably accurately by some preliminary simulations then perform one long simulation of about $5 \times 10^{6} \mathrm{MC}$ steps to obtain reasonable statistics. Then one extrapolate the data to $\beta_{c}(L)$ to measure $\Delta F_{E}(L)$. In practice we also smoothed the data by an eighth-order polynomial fit.

Fairly extensive testing of temperature driven first order transitions was carried out for Potts models in two and three dimensions. Sizes were limited to $L \leq 60$ for $d=2$ and $L \leq 14$ for $d=3$ by the cornputer time available since good statistics are much more important than large system sizes. Despite $L / \xi<0.05$ for $q=5$, the first order nature was unambiguously shown for $q=5,6,8,10$ 


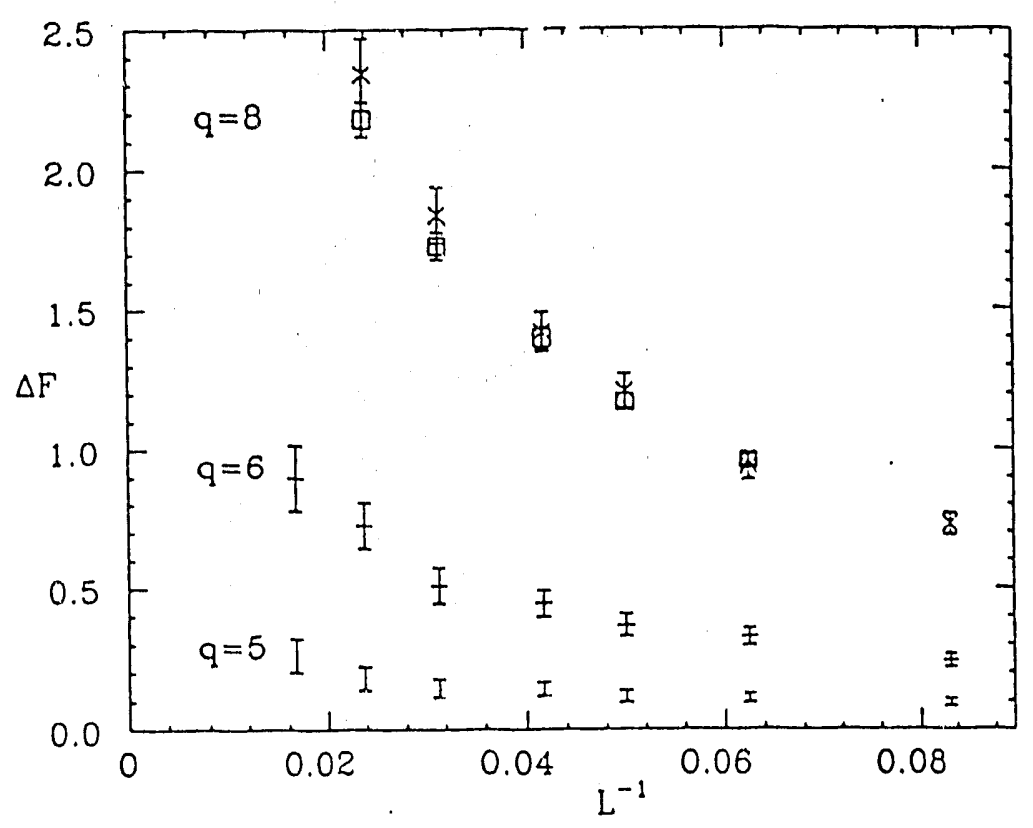

Fig. 2. Peak height $\Delta F_{E}(L)$ for $q=5,6,8$ Potts models. Open squares for $q=8$ were obtained by Swendsen-Wang algorithm ${ }^{19}$; all others by Metropolis algorithm.

12,14 Poits models (see figures 1 and 2). From three dimensional Potts model with $L \leq 14$ we find ${ }^{20}$, for the first time by simulation, $q_{c}=2.45 \pm 0.1$. We also conclude that there is no essential singularity in correlation length or latent heat near $q=q_{c}$ in contrast to two dimensions.

\section{FIELD DRIVEN FIRST ORDER TRANSITIONS}

When applied to a continuous transition, the general method described in previous sections provides an easy and surprisingly accurate way of calculating the correlation length exponent $\nu$, critical temperature $T_{c}$ and somewhat less accurate order parameter exponent $\beta / \nu$. Test ${ }^{14}$ on Ising model in two dimensions up to $L \leq 60$ gives $\nu=1.003(10), 2 \beta / \nu=0.247(8)$. We get $\nu=0.634(6), 2 \beta / \nu=1.02(3)$ in three dimensions with $L \leq 14$. Here we describe, somewhat in details, direct application of the general method to $q=3$ ferromagnetic Potts model in two dimensions to obtain $T_{c}$ and $1 / \nu$.

Let's consider a square lattice of $L \times L=N$ with periodic boundary conditions. If we denote $N_{i}$ as the number of spins with $q=i$, we have a order parameter vector $\vec{N}=\left(N_{1}, N_{2}, N_{3}\right)$ and $N=N_{1}+N_{2}+N_{3}$ with two degrees of freedom. We want rewrite $\vec{N}$ in terms of two basis vectors $\vec{a}=1 / \sqrt{6}(2,-1,-\cdot 1)$ and $\vec{b}=1 / \sqrt{2}(0,1,-1)$ which are perpendicular to $(N, N, N) / 3$ as follows

$$
\vec{N}=(N, N, N) / 3+N_{a} \vec{a}+N_{b} \vec{b}
$$




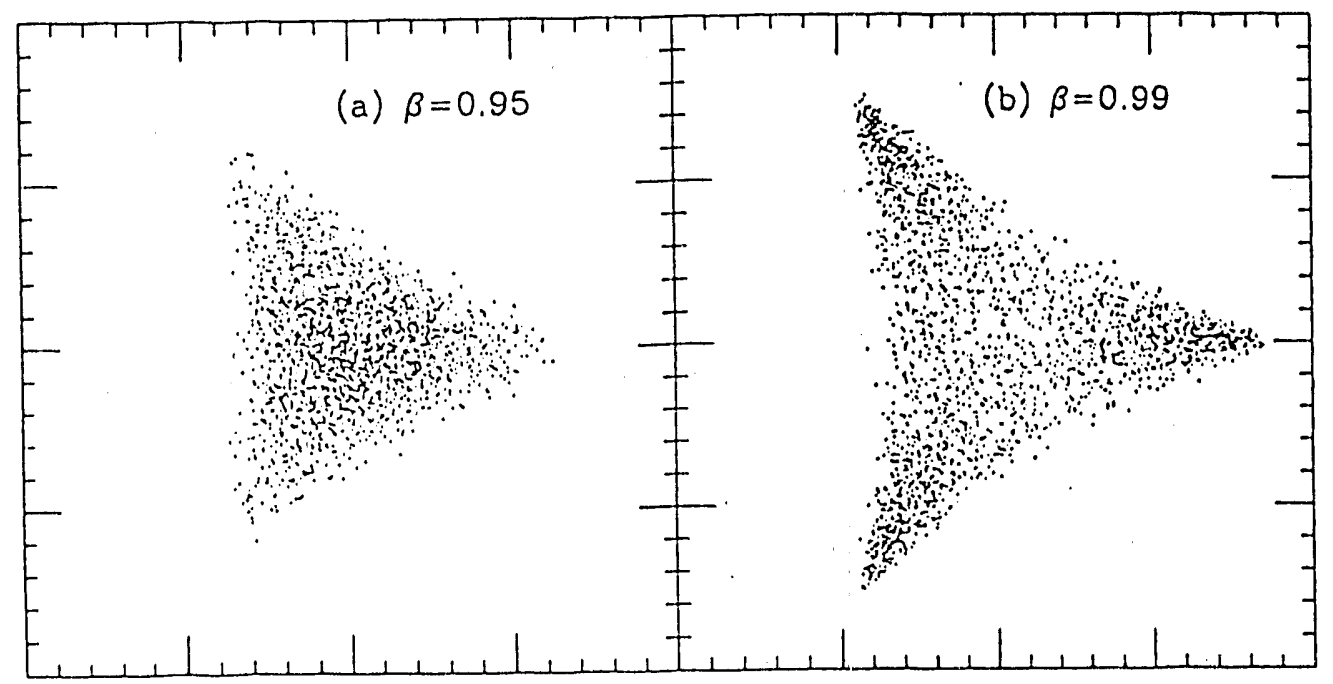

Fig. 3. Scatter plot of order parameter of $g=3$ Potts model for $30 \times 30$.

where $N_{a}=\left(2 N_{1}-N_{2}-N_{3}\right) / \sqrt{6}$ and $N_{b}=\left(N_{2}-N_{3}\right) / \sqrt{2}$. We take the order parameter as the last two terms in Eq. 8

In this way the scatter plot of the order paranneter for $N=30 \times 30$ is shown in Fig. 3. For $\beta=0.99$, one can clearly see the three dense areas corresponding to states where one of $q$ is dominating the others. In the deconfinement transition $^{21,22}$ of quarks in $S U(3)$, one obtain similar distributions of order parameter from the Lattice QCD simulations. A criterion based on the certain ratio of occurrence between the states of $\theta=0$ and $\pi / 3$ is often used ${ }^{22}$ to determine the nature of transition and critical temperature. But one has to be very careful using this kind of criterion since direct application of it to this model would lead to $\beta_{c} \simeq 0.97$ with first order transition. The three dense areas in Fig. 3. is only an artifact of finite size system (we know that the model undergoes a second order transition at $\beta=\ln (1+\sqrt{3}) \simeq 1.005$ ), and they will disappear with larger system size just as the case of $\beta=0.95$.

Near phase transition the free energy as a function of order parameter can be written in terms of scaling variables $x_{i}=\left(N_{i}-N / 3\right) L^{\beta / \nu}$ and $y=t L^{1 / \nu}$ with $x_{1}+x_{2}+x_{3}=0$. The symmetry of the system allows that the free energy can be expanded only by two terms of $x_{1}^{2}+x_{2}^{2}+x_{3}^{2}$ and $x_{1} x_{2} x_{3}$. With some algebra one can show that up to $O\left(r^{4}\right)$

$$
F(r, \theta, y)=A r^{2}+B r^{3} \cos \theta\left(4 \cos ^{2} \theta-3\right)+C r^{4}+D y r^{2} \cdots
$$

where $(r, \theta)$ comes in as the polar coordinates of $x_{i}$ s. We find $\Delta F$ defined as the difference between minima $(\theta=0, \pm 2 \pi / 3)$ and saddle points $(\theta=\pi, \pm \pi / 3)$ reads just same in the form of Eq. 5. A plot of $\Delta F_{M}(L, t)$ is shown in Fig. 4 for $10 \leq L \leq 36$. A very similar structure is seen for Ising models in two and three dimensions. ${ }^{14}$ 


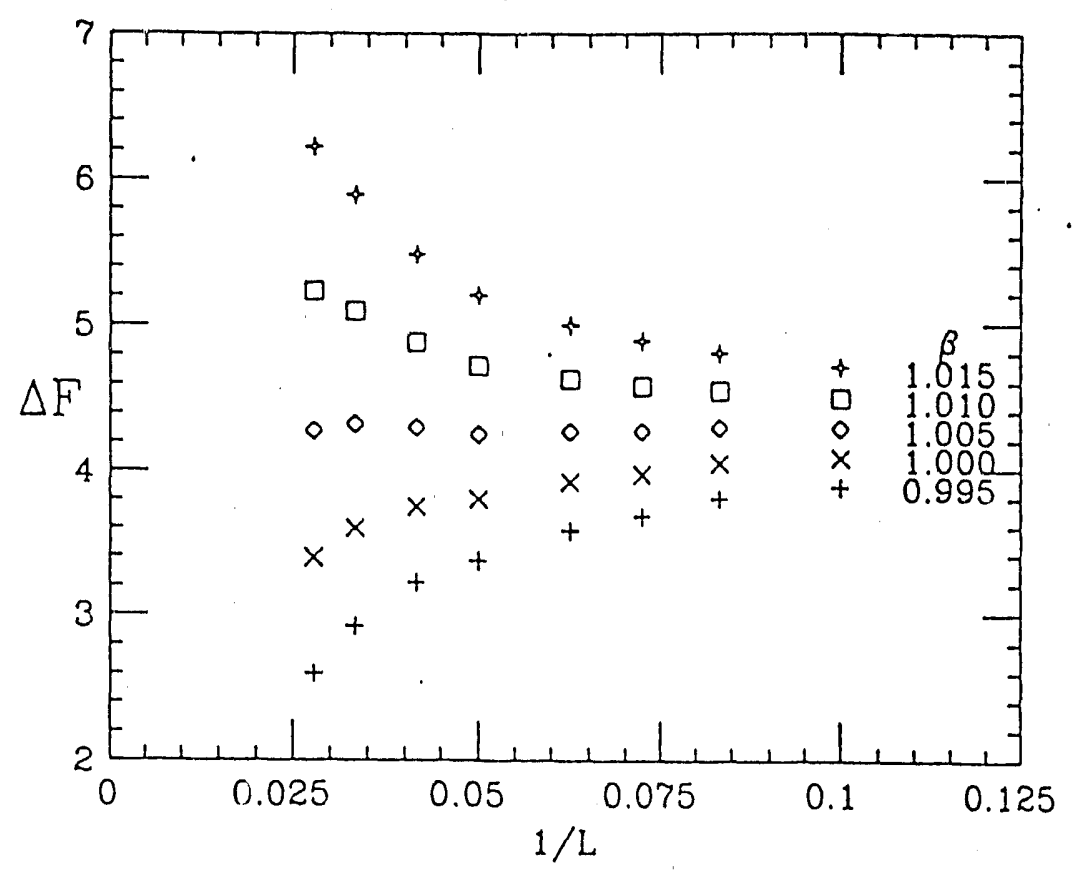

Fig. 4. $\Delta F_{M}(\beta, L)$ near second order transition of $q=3, d=2$ Potts model. All points at fixed $L$ are obtained from $\beta=1.005$ by extrapolation

With Eq. 5 the temperature derivative

$$
S(L) \equiv \delta \Delta F(t, L) / \delta t \sim L^{1 / \nu}
$$

provides a numerical way of obtaining $1 / \nu$ from a linear fit of $\ln S$ and $\ln L$ without exact value of $T_{c}$. This is very important since $T_{c}$ and $1 / \nu$ are obtained by independent two separate ways. For the case of Ising models $S(L)$ was obtained by a numerical derivative using the histogram method ${ }^{18}$ mentioned earlier. For $q=3$ model, observation that the maxima and saddle points occur only along the symmetry axis of $\theta=n \pi / 3$ with $n=1, \cdots, 6$ enables us to get $S(L)$ from $^{23}$

$$
S(L)=<E>_{0}-<E>_{\pi / 3}
$$

where $\left\langle E>_{0, \pi / 3}\right.$ is the average internal energy for the symmetry axis of $\theta=$ $0, \pi / 3$.

We show $S(L)$ in Fig. 5 for $5 \leq L \leq 36$ where we find $1 / \nu=1.19 \pm 0.02$ taking large sizes $(L \geq 12)$. The order parameter exponent $\beta / \nu$ can be obtained by measuring the separation between minima (or saddle points) and the origin at $T_{c}$ which scales as $L^{-\beta / \nu}$. The major source of error for $\beta / \nu$ is from the small uncertainty in $T_{c}$. Once $T_{c}$ is iound, simulations in microcanonical ensemble along the symmetry axes will be more effective to find exponents.

The method has been applied to simple models with great success but a note of caution is necessary here. First it is very important to know that the 


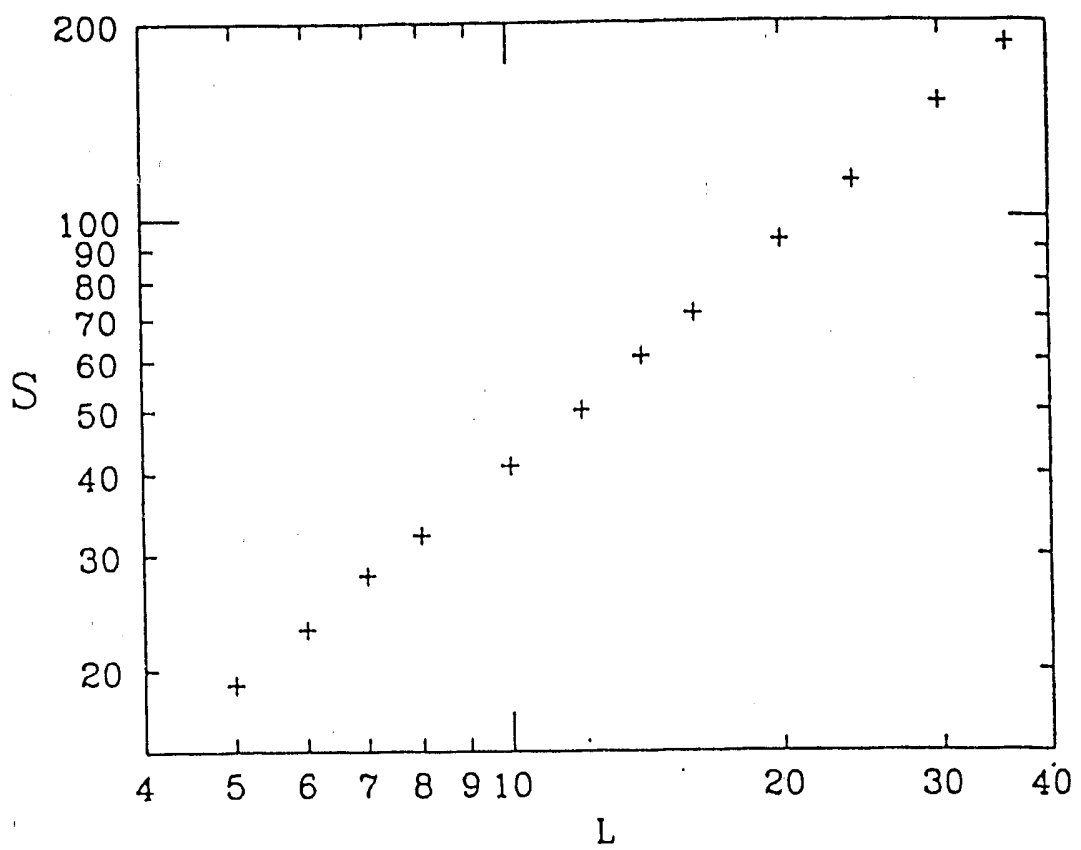

Fig. 5. $S(L) \sim L^{1 / \nu}$ for $5 \leq L \leq 36$. Note curvature for $L \leq 12$ indicating an irrelevant variable.

transition is continuous so this kind of approach can give a very good estimates of exponents. Secondly, the presence of slowly decaying irrelevant variables may effect the estimates. For the case of $q=3$ Potts model in two dimensions we observe small upward curvature in $S(L)$ for $L \leq 12$

\section{MORE APPLICATIONS}

The original motivation for this general method was to study the problem of fully frustrated Josephsen junction arrays which is a two-dimensional regular periodic array of superconducting grains in a perpendicular magnetic field of half flux quantum per plaquette. This has been intensively studied ${ }^{24}$ for many years both experimeritally and theoretically with rather inconclusive results. Here we leave the results of the direct application of the method to references 25-27.

The method also has beer applied to the study of Hopfield network with limited-connectivity. ${ }^{28}$ Two-dimensional melting study of hard disk system with this method shows ${ }^{29}$ that it is of first order transition unambiguously. Also some thermodynamic quantities such as the critical pressure and correlation length is obtained. Applications to other melting systems are underway

\section{CONCLUSION AND DISCUSSION}

We have developed a remarkably powerful method for numerically detecting first order transitions since the qualitative behavior of $\Delta F(L)$ is the characteristic of the type of transition. At a continuous transition gocd estimates of the 
critical exponents and critical temperature can be obtained as a by product. Extensive tests were carried out to known systems showing the power and the simpleness of the method. Applications to many other systems bring out new results $^{20,25-29}$ as mentioned in the text.

Numerical proof of the second order phase transition ( $q=3.9$ Potts model in two dimensions, for example) is more difficult subject especially when $a=0$ in Eq. 5, which seems to be the most of the cases we tested. The success depends on how one can increase $a$ without affecting the critical properties of the system. One candidate which is under investigation, is using the effect of dimensional crossover.

There are lots of rooms here one can investigate more on the method not to mention on the application side. A theory for $A(E, M, \beta, L)$ near a tricritical point would be very helpful since it is notoriously difficult to locate by numerical methods. We have not yet studied systems with a continuous symmetry such as $O(n)$ model. This kind of study will help to understand, for example, the problem of general two-dimensional melting. With growing interest in flux line lattice in HTC superconductors, this method should, in principle, give the best out of the numerical simulations

This work was supported by the NSF grant no. DMR-8918358 (JMK and JL), by US Department of Energy under contract no. W-31-109-ENG-3B (JL).

\section{REFERENCES}

1. M. N. Barber, Phase Transitions and Critical Phenomena, edited by C. Domb and J. L. Lebowitz (Academic, New York, 1983), Vol. 8, p.145.

2. V. Privman and M. E. Fisher, J. Stat. Phys. 33, 385 (1983); Phys. Rev. B 32, 447 (1985).

3. K. Binder, Rep. Prog. Phys. bf 50,783 (1987).

4. P. Peczak and D. P. Landau, Phys. Rev. B 39,11932 (1989).

5. J. F. McCarthy, Phys. Rev. B 41,9530 (1990)

6. M. Fukugita, H. Mino, M. Okawa and A. Ukawa, J. Phys. A23, L561 (1990).

7. R. B. Potts, Proc. Camb. Phil. Soc. 48,106 (1952); F. Y. Wu, Rev. Mod. Phys. 54,235 (1982).

8. K. Binder, Monte Carlo Method in Statistical Physics, edited by K. Binder, Topics in Current Physics, (Springer, Berlin, Heidelberg and New York, 1979) Vol. 7, p.1.

9. D. P. Landau and K. Binder, Phys. Rev. B 17, 2328 (1978).

10. K. Binder and D. P. Landau, Phys. Rev. B 21, 1941 (1980).

11. K. Binder, Phys. Rev. Lett. 47, 693 (1981); Z. Phys. B 43, 119 (1981).

12. J. Lee and J. M. Kosterlitz, Phys. Rev. B 43, 3265 (1991).

13. M. S. S. Challa, D. P. Landau, K. Binder, Phys. Rev, B 34, 184 (1986).

14. J. Lee and J. M. Kosterlitz, Phys. Rev. Lett. 65, 137 (1990).

15. K. Binder, Rep. Prog. Phys. 50, 783 (1987). 
16. For recent reviews see Finite Size Scaling and Numerical Simulations of Statistical Systems, edited by V. Privman (World Scientific, Singapore, 1990).

17. E. Eisenriegler and R. Tomaschitz, Phys. Rev. B 35, 4876 (1987).

18. A. M. Ferrenberg and R. H. Swendsen, Phys. Rev. Lett. 61, 2635 (1988).

19. R. H. Swendsen and J-S. Wang, Phys. Rev. Lett. 58, 86 (1987).

20. J. Lee and J. M. Kosterlitz, Phys. Rev. B 43, 1268 (1991).

21. A. D. Kennedy, et al., Phys. Rev. Lett. 54, 87 (1985); S. A. Gottlieb, et al., ibid., 55, 1958 (1985); M. Fukugita, M. Okawa and A. Ukawa, Nucl. Phys. B 337, 181 (1990).

22. N. H. Christ and A. E. Terrano, Phys. Rev. Lett. 56, 111 (1986); N. H. Christ and H.-Q. Ding, ibid., 60,1367 (1988).

23. J. Lee, Ph. D. Thesis (unpublished).

24. S. Teitel and C. Jajaprakash, Phys. Rev. Lett. 51, 199 (1983); W. Y. Shih and D. Stroud, Phys. Rev. B 32, 158 (1985).

25. E. Granato, J. M. Kosterlitz, J. Lee and P. M. Nightingale, Phys. Rev. Lett. 66, 1090 (1991)

26. J. Lee, J. M. Kosterlitz and E. Granato, Phys. Rev. B 43, 11531 (1991)

27. J. Lee, E. Granato and J. K. Kosterlitz, Phys. Rev. B (in press).

28. K. J. Strandburg, M. Peshkin and D. F. Boyd (unpublished).

29. J. Lee (unpublished). 
DATE

FILMED

$3 / 3 / 92$ 
